\title{
Effect of dietary conjugated linoleic acid isomers on water and glycerol permeability of kidney membranes
}

\author{
Graça Soveral $^{\mathrm{a}, \mathrm{b}, *}$, Ana P. Martins ${ }^{\mathrm{a}}$, Susana V. Martins ${ }^{c}$, Paula A. Lopes ${ }^{c}$, Cristina M. Alfaia ${ }^{\mathrm{c}}$, \\ José A.M. Prates ${ }^{c}$, Teresa F. Moura ${ }^{a}$ \\ ${ }^{a}$ REQUIMTE, Dep. Química, FCT-UNL, 2829-516 Caparica, Portugal \\ ${ }^{\mathrm{b}}$ Faculdade de Farmácia, Universidade de Lisboa, 1649-003 Lisboa, Portugal

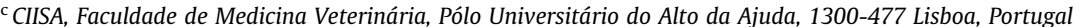

\section{A R T I C L E I N F O}

Article history:

Received 14 March 2009

Available online $\mathrm{xxxx}$

\section{Keywords:}

Conjugated linoleic acid

Water permeability

Glycerol permeability

Kidney

Membrane vesicles

Aquaporin

\begin{abstract}
A B S T R A C T
Conjugated linoleic acid (CLA) refers to a group of positional and geometrical isomers of linoleic acid in which the double bonds are conjugated. Dietary CLA has been associated with various health benefits although details of its molecular mode of action remain elusive. The effect of CLA supplemented to palm oil-based diets in Wistar rats, as a mixture of both or isolated $c 9, t 11$ and $t 10, c 12$ isomers, was examined on water and glycerol membrane permeability of kidney proximal tubule. Although water permeability was unaltered, an increase in glycerol permeability was obtained for the group supplemented with CLA mixture, even though the activation energy for glycerol permeation remained high. This effect was correlated with an increased CLA isomeric membrane incorporation for the same dietary group. These results suggest that diet supplementation with CLA mixture, in contrast to its individual isomers, may enhance membrane fluidity subsequently raising kidney glycerol reabsorption.
\end{abstract}

(C) 2009 Published by Elsevier Inc.

\section{Indroduction}

Conjugated linoleic acid (CLA) refers to a multiplicity of geometrical and positional isomers of linoleic acid (C18:2n-6) with conjugated double bonds. These double bonds, in either trans $(t)$ or cis $(c)$ configuration, are present predominantly from positions 6,812,14 . Although being a natural dietary fatty acid from ruminant origin, CLA has been associated with some health benefits and, for this reason, has attracted much scientific attention. A growing number of experimental studies using laboratory animals, as well as human and cell culture systems, suggests that $c 9, t 11$ and $t 10, c 12$ CLA isomers may prevent heart disease, diabetes and atherosclerosis, affect weight control and inhibit the growth of various types of cancer [1]. The most abundant CLA isomer in ruminant foods is the $c 9, t 11$ [2], produced in the rumen by biohydrogenation of dietary C18 polyunsaturated fatty acids (PUFA) and in the tissues through delta9 desaturation of $C 18: 1 t 11$, although nutritional supplements posses a mixture of equal amounts of $c 9, t 11$ and $t 10, c 12$ isomers. These commercial CLA preparations industrially produced are attracting consumers' interest due to the purported body fat-lowering effects of CLA, coupled to the perception of a 'natural' compound. However, the attempts to replicate these beneficial findings in humans have produced inconsistent results $[3,4]$.

\footnotetext{
* Corresponding author. Address: REQUIMTE, Dep. Química, FCT-UNL, 2829-516 Caparica, Portugal. Fax: +351 212948550

E-mail addresses: gsoveral@ff.ul.pt, soveral@dq.fct.unl.pt (G. Soveral).
}

Albeit several candidate mechanisms including alterations in membrane structure and composition, signal transduction, gene expression and immunity have been suggested as future research directions [5], the metabolic pathways by which CLA isomers elicit their effects on body composition remain largely unknown.

In the kidney, at least seven aquaporins are expressed at different sites along the nephron. The orthodox aquaporin-1 (AQP1) is extremely abundant in the proximal tubule and descending limb where it appears to be the main site for proximal tubule water reabsorption. The other aquaporin isoform abundantly expressed at proximal tubule brush border membrane is AQP7, an aquaglyceroporin which was also found in other tissues like testis and adipocytes [6,7]. In the kidney, AQP7 plays a minor role in water transport but constitutes a major glycerol-reabsorbing pathway preventing glycerol from being excreted into urine [8]. Besides molecular biology approaches that include aquaporin gene deletion and overexpression, evaluation of the osmotic permeability coefficient $\left(P_{f}\right)$ and Arrhenius activation energy $\left(E_{a}\right)$ are among the few experimentally measurable parameters that indicate the contribution of AQPs to water transport, thus playing an important role in the debate on the physiological function of specific AQPs and their regulation. As for glycerol transport, fewer studies have been performed on kidney apical membranes glycerol permeability $\left(P_{g l y}\right)$. The only reported data has been taken from measuring the serum and urine glycerol levels in wild-type and AQP7 knockout mice [8]. Despite being under attention by the research community 
during the last decades, no studies have been published addressing CLA isomers effects on kidney water and glycerol reabsorption.

In this study, the effect of dietary CLA isomers on both water and glycerol permeabilities of kidney proximal tubule membranes were investigated. The preparation of isolated brush-border membrane vesicles (BBMV) provides the possibility of studying transport processes independently of other cellular events. Moreover, being more resistant and viable than intact cells, vesicles can be prepared with any chosen internal media, expanding the range of experimental conditions. Four groups of Wistar rats were fed saturated diets, formulated to mimic patterns of typical human diets from the Western societies [9] and supplemented or not with $c 9, t 11$ and $t 10, c 12$ CLA isomers in separate or as a mixture. Using purified kidney BBMV preparations, membrane permeabilities $P_{f}$ and $P_{g l y}$ were assessed by stopped-flow light scatter. The $E_{a}$ for water and glycerol transport was calculated. Additionally, the fatty acid profile of membrane vesicles was determined in order to assess CLA isomers incorporation level into total membrane lipids.

\section{Materials and methods}

Experimental design: animals and diets. The experimental protocol of this study was reviewed by the ethics commission of CIISA/ FMV and approved by the Animal Care Committee of the National Veterinary Authority following the appropriated European Union guidelines (N. 86/609/EEC). Wistar male rats $(n=39$, Harlan Interfauna Iberica, S.L., Barcelona, Spain) with an initial body weight of $114.7 \pm 0.98 \mathrm{~g}$ (mean \pm SEM) were acclimatized and fed a standard diet (Harlan Teklad Global Diets ${ }^{\odot} 2014$ ) without CLA for 1 week. After this period, rats were allocated to four groups of ten animals each. The standard pellets (Harlan Teklad Global Diets ${ }^{\circledR}$ 2014) were enriched with palm, sunflower and CLA oils as described [10]. Briefly, a 5\% palm oil-based diet was supplemented with CLA oils to reach $1 \%$ of CLA isomers $(0.5 \%$ of $\mathrm{c} 9, \mathrm{t} 11$ and $0.5 \%$ of $\mathrm{t} 10, \mathrm{c} 12)$ for the mixture group $\mathrm{M}$ and $0.5 \%$ of $\mathrm{c} 9, \mathrm{t} 11$ or $0.5 \%$ of $\mathrm{t} 10, \mathrm{c} 12$ isomers for groups $C$ and $\mathrm{T}$, respectively. A control group with $5 \%$ of palm oil and without CLA was included for comparative proposes, The diets were provided ad libitum during 8 weeks and, after a $12 \mathrm{~h}$ fast, rats were euthanized by decapitation, under light inhalation anesthesia (isofluorane, Abbott, IL, USA). The kidneys were ex-

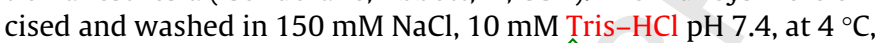
for subsequent preparation of BBMV.

Preparation of brush-border membrane vesicles. BBMV were prepared from rat renal cortex as described [11]. After kidney decapsulation, the whole process was conducted at $4{ }^{\circ} \mathrm{C}$ in the presence of a single buffer containing $100 \mathrm{mM}$ mannitol, $10 \mathrm{mM}$ Tris-Hepes $\mathrm{pH}$ 7.4. Kidney cortexes from each dietary group were pooled to give one final vesicle preparation. Prior to osmotic experiments, vesicles were resuspended and homogenized in the same buffer but where mannitol was replaced by cellobiose (final osmolarity $120 \mathrm{mOsM}$ ), a solute shown to be highly impermeant over a larger time scale [12]. The membrane preparations obtained were either immediately used for experiments or stored in liquid nitrogen for later use. Protein content was determined by the Bradford technique [13], using bovine albumin as standard.

Osmolarity measurements. All solution osmolarities were determined from freezing point depression on a semi-micro osmometer (Knauer GmbH, Germany). Standards of 100 and $400 \mathrm{mOsM}$ were analyzed prior to samples, which were measured in triplicate.

Vesicle size determination. Vesicle size of all the membrane preparations was determined in isosmotic conditions by the QuasiElastic Light Scattering (QELS) technique (Brookhaven Instruments BI-90). Application of this technique to vesicular size measurements have been published [14].
Stopped-flow experiments on water and glycerol permeability. Stopped-flow experiments were performed on a HI-TECH Scientific PQ/SF-53 apparatus, which has a 2 ms dead time, temperature controlled, interfaced with an IBM PC/AT compatible 80386 microcomputer. Experiments were performed at different temperatures. Typically, five runs were usually stored and analyzed in each experimental condition. For the measurement of osmotic water permeability, $0.1 \mathrm{ml}$ of vesicles $(0.4 \mathrm{mg}$ protein $/ \mathrm{ml})$ ressuspended in cellobiose buffer was mixed with an equal amount of isoosmotic (120 mOsM) or hyperosmotic (180 mOsM) cellobiose solutions to reach an inwardly directed gradient of solute. The kinetics of vesicle shrinkage were measured from the time course of $90^{\circ}$ scattered light intensity at $400 \mathrm{~nm}$ until a stable light scatter signal was attained. The osmotic water permeability coefficient was estimated by fitting the light scatter signal to a single exponential curve and using the linear relation between $P_{f}$ and the exponential time constant $k$ [15], $P_{f}=k\left(V_{\text {oo }} / \mathrm{A}\right)\left(1 / V_{w}\left(\text { osm }_{\text {out }}\right)_{\infty}\right)$, where $V_{w}$ is the molar volume of water, $V_{o} / A$ is the initial volume to area ratio of the vesicle preparation, and $\left(\alpha s m_{\text {out }}\right)_{\infty}$ is the final medium osmolarity after the applied osmotic gradient. For glycerol permeability, vesicles equilibrated in 120 mOsM cellobiose buffer were confronted to an external solution where the impermeant cellobiose was partially substituted with glycerol (60 mOsM cellobiose, 60 mOsM glycerol, creating an inwardly directed glycerol gradient with no osmotic shock). Glycerol influx in response to its chemical gradient was followed by water influx with subsequent vesicle swelling. Glycerol permeability was calculated as $P_{g l y}=k\left(V_{o} / A\right)$, where $k$ is the single exponential time constant fitted to the light scattering time course corresponding to glycerol influx [16].

Activation energy calculation. Water and glycerol permeabilities were measured at five different temperatures between $7^{\circ} \mathrm{C}$ and $37^{\circ} \mathrm{C}$. The $E_{a}$ of water and of glycerol transport was evaluated from the slope of the Arrhenius plot $\left(\ln P_{f}\right.$ or $\ln P_{g l y}$ as a function of $\left.1 / T\right)$ multiplied by the gas constant $R$.

Fatty acid profile. The fatty acid profile of BBMV preparations, resulting from pooled kidney cortexes for each dietary group, was obtained using the transesterification method [17]. The fatty acid methyl esters were analyzed through single injections by gas chromatography (GC) and methyl esters of CLA isomers were individually separated by high performance liquid chromatography (HPLC). The percentage of CLA isomers was calculated from their HPLC areas relative to the area of the main isomer $c 9, t 11$ identified by GC [18].

Statistics. Data are expressed as mean \pm SD. Values of permeability coefficients were analyzed and compared with control using one-way ANOVA followed by Student's $t$ test. Differences with $P<0.05$ were considered significant.

\section{Results}

Characterization of BBMV from kidney proximal tubule

BBMV prepared from rat kidney cortex by differential centrifugation showed an enrichment in enzyme specific activity (BBMV/ crude homogenate) of the apical markers leucine-aminopeptidase [19] and alkaline phosphatase [20], as well as the basolateral markers $\mathrm{Na}^{+} / \mathrm{K}^{+}$ATPase [21] and $\mathrm{K}^{+}$stimulated phosphatase [22], assayed as described, of $13.2 \pm 1.7,9.6 \pm 1.5,1.1 \pm 0.04$ and $0.4 \pm 0.05$ $(n=20)$, respectively.

Vesicle size of all prepared batches of vesicles determined by QELS revealed homogeneous populations, showing unimodal distributions with a mean diameter of $415 \pm 35 \mathrm{~nm}(n=26)$. These results assure purified and homogeneous BBMV preparations. 
Glycerol but not water permeability is increased in the CLA mixture group

To assess the effect of dietary CLA isomers on water membrane permeability, we measured the $P_{f}$ of purified BBMV. The osmotic challenges were performed at different temperatures using the stopped-flow technique. Fig. 1A shows a typical time course of volume change induced by osmotic shocks of $-60,0$ and $60 \mathrm{mOsM}$ cellobiose, carried out with the control group membrane vesicles at $23{ }^{\circ} \mathrm{C}$. BBMV were osmotically responsive when confronted to hypo- and hyper-osmotic shocks of impermeant solute, as can be seen by the light scatter traces reflecting volume changes. Averaged $P_{f}$ was $(13.86 \pm 2.11) \times 10_{\wedge}^{-3} \mathrm{~cm} \mathrm{~s}_{\wedge}^{-1}$. Glycerol transport was studied in membrane vesicles prepared from control and CLA groups submitted to an inward $60 \mathrm{mOsM}$ glycerol gradient in isoosmotic conditions, in order to avoid initial water movements. As glycerol enters, vesicles progressively swell till they reach an equilibrium volume, as shown in Fig. 1B, and the rate constants of glycerol influx were used for $P_{g l y}$ evaluation. Averaged $P_{g l y}$ for the control group was $(2.55 \pm 0.63) \times 10_{\wedge}^{-6} \mathrm{~cm} \mathrm{~s}_{\wedge}^{-1}$. Fig. 2 shows the permeability results obtained for the dietary groups at $23{ }^{\circ} \mathrm{C}$, for water (panel A) and glycerol (panel B). It can be observed that the values of osmotic water permeability $P_{f}$ are within the same range with no significant differences among groups $(P>0.05$, Fig. 2A). As for glycerol, a relevant increase in $P_{g l y}$ to roughly twofold the control $(P<0.05)$ was obtained for the mixed isomers (group $\mathrm{M}$ ) at all temperatures tested. However, the isolated $c 9, t 11$ CLA isomer (group C) did not show any significant change compared with the control, and the $t 10, c 12$ (group T) showed only a slight decrease of $P_{g l y}(P>0.05$, Fig. 2B).
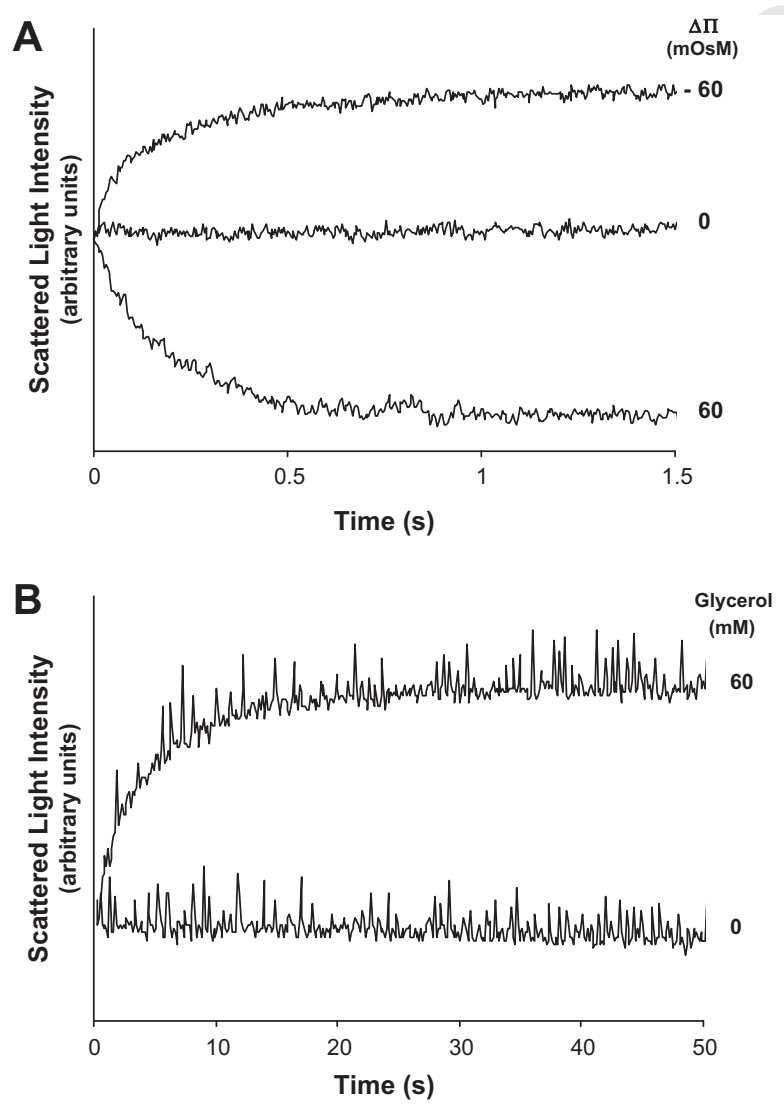

Fig. 1. Representative plots of brush border membrane permeability to water (A) (osmotic shocks of $-60,0$ and 60 mOsM cellobiose gradients) and glycerol (B) (isosmotic shock, $60 \mathrm{mOsM}$ glycerol gradient), by stopped-flow light scattering.
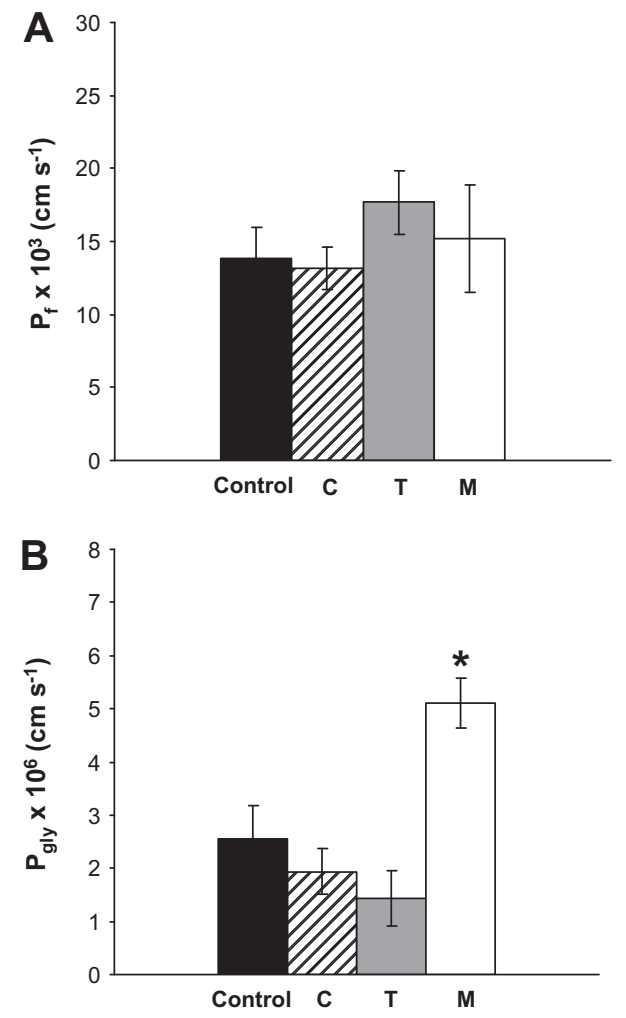

Fig. 2. Water (A) and glycerol (B) permeability coefficients obtained at $23^{\circ} \mathrm{C}$ for the different membrane vesicles. Values are mean \pm SD of triplicates of at least three independent experiments $(n=9)$. " Different from control, $P<0.05$.

Activation energy for water and glycerol transport is unchanged among groups

Fig. 3 depicts the $E_{a}$ values for both glycerol and water transport. The $E_{a}$ for glycerol transport was similar among groups $(P>0.05)$ being always very high, above $17.6 \mathrm{kcal} \mathrm{mol}^{-1}$ $\left(73.7 \mathrm{~kJ} \mathrm{~mol}^{-1}\right)$. Conversely, the $E_{a}$ obtained for water transport was low for any group tested, between 4.4 and $4.5 \mathrm{kcal} \mathrm{mol}^{-1}$ (18.4 and $18.8 \mathrm{~kJ} \mathrm{~mol}^{-1}$ ) but still not different among groups $(P>0.05)$.

\section{CLA membrane incorporation is enhanced in the mixture group}

The fatty acid composition of BBMV from the four dietary groups was particularly different for CLA isomers percentages, as shown in Table 1 . The deposition of total saturated fatty acids

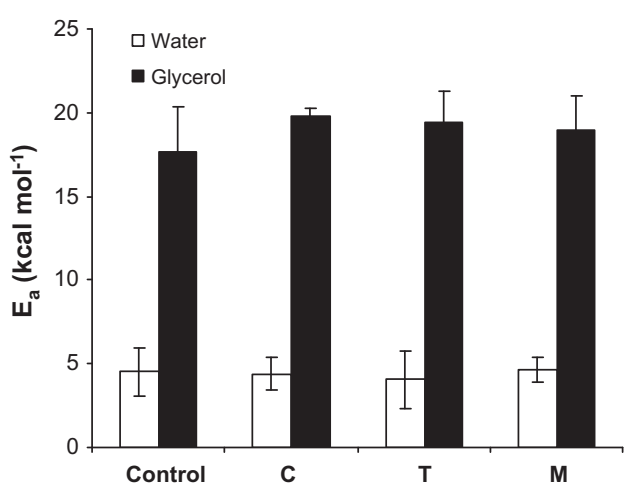

Fig. 3. Activation energy of water (empty bars) and glycerol (full bars) for the dietary groups tested. Values are mean \pm SD $(n=3)$. No significant differences between the dietary CLA groups and control within each type of permeability were detected $(P>0.05)$. 

and glycerol permeability of kidney proximal tubule membrane vesicles, from rats fed CLA on a diet supplemented with a mixture

Table 1

Fatty acid profile (\% fatty acids) of BBMV for the different dietary groups.

\begin{tabular}{|c|c|c|c|c|}
\hline & \multicolumn{4}{|c|}{ Dietary groups } \\
\hline & Control & $\mathrm{C}$ & $\mathrm{T}$ & M \\
\hline C16:0 & 21.40 & 20.36 & 21.75 & 20.79 \\
\hline C17:0 & 0.22 & 0.23 & 0.24 & 0.26 \\
\hline C18:0 & 19.30 & 20.86 & 21.99 & 22.41 \\
\hline C20:0 & 0.17 & 0.08 & 0.08 & 0.11 \\
\hline $\mathrm{C} 22: 0$ & 0.09 & 0.19 & 0.33 & 0.43 \\
\hline $\mathrm{C} 24: 0$ & 0.00 & 0.10 & 0.08 & 0.18 \\
\hline$\Sigma \mathrm{SFA}$ & 41.18 & 41.83 & 44.47 & 44.18 \\
\hline C16:1c9 & 0.98 & 0.35 & 0.26 & 0.41 \\
\hline C18:1c9 & 6.79 & 6.65 & 6.57 & 6.76 \\
\hline C18:1c11 & 3.05 & 2.05 & 1.73 & 1.90 \\
\hline C20:1c11 & 0.11 & 0.10 & 0.10 & 0.17 \\
\hline$\Sigma$ MUFA & 10.93 & 9.15 & 8.66 & 9.25 \\
\hline$C 18: 2 n-6$ & 11.74 & 10.69 & 11.78 & 11.94 \\
\hline$C 18: 3 n-3$ & 0.14 & 0.05 & 0.05 & 0.15 \\
\hline$C 20: 2 n-6$ & 0.27 & 0.30 & 0.30 & 0.16 \\
\hline$C 20: 3 n-3$ & 0.00 & 0.07 & 0.09 & 0.23 \\
\hline$C 20: 3 n-6$ & 1.22 & 0.61 & 0.64 & 0.64 \\
\hline$C 20: 4 n-6$ & 31.18 & 34.24 & 31.15 & 30.07 \\
\hline$C 20: 5 n-3$ & 0.12 & 0.07 & 0.08 & 0.12 \\
\hline$C 22: 4 n-6$ & 0.81 & 0.65 & 0.72 & 0.59 \\
\hline$C 22: 5 n-3$ & 0.25 & 0.23 & 0.27 & 0.45 \\
\hline$C 22: 6 n-3$ & 2.16 & 1.89 & 1.72 & 1.75 \\
\hline$\Sigma$ PUFA & 47.89 & 48.81 & 46.81 & 46.10 \\
\hline $\mathrm{C} 18: 2 t 7, c 9$ & n.d. & 0.011 & 0.011 & 0.038 \\
\hline $\mathrm{C} 18: 2 c 9, t 11$ & n.d. & 0.181 & 0.003 & 0.220 \\
\hline $\mathrm{C} 18: 2 t 10, c 12$ & n.d. & 0.002 & 0.029 & 0.105 \\
\hline $\mathrm{C} 18: 2 c / t 11,13$ & n.d. & 0.005 & n.d. & 0.016 \\
\hline $\mathrm{C} 18: 2 c / t \quad 12,14$ & n.d. & 0.004 & 0.003 & 0.027 \\
\hline$\Sigma \mathrm{C} 18: 2 t, t$ & n.d. & 0.015 & 0.008 & 0.065 \\
\hline$\Sigma$ CLA & n.d. & 0.219 & 0.054 & 0.471 \\
\hline
\end{tabular}

n.d., not detected.

$\Sigma$ SFA, sum of saturated fatty acids; $\Sigma$ MUFA, sum of monounsaturated fatty acids; $\Sigma$ PUFA, sum of polyunsaturated fatty acids; $\Sigma$ CLA, sum of conjugated linoleic acid isomers. of $c 9, t 11$ and $t 10, c 12$ isomers, or both in separate. Since commercial CLA is composed by a mixture of two isomers, it is of interest to determine which is responsible for biological activity through possible modulation on cell membrane permeability. The use of isolated BBMV offers an experimental system that allows the composite permeability of epithelial cells to be split into its component parts and yields more precise information about driving forces of transport in epithelia.

In this study, CLA did not affect water transport through the vesicle membrane, neither as a mixture nor using both isomers in separate. In fact, the low $E_{a}$ obtained is compatible with channel mediated water fluxes [23], independently from the tested group. Regarding the $E_{a}$ for glycerol, the strong temperature dependence revealed by the high value obtained suggests lipid-rather than channel-mediated glycerol transport [24]. This result may indicate that the aquaglyceroporin AQP7 was not functional or that its level of expression/incorporation in the brush border membrane was very low, resulting in a main contribution of the lipid pathway to glycerol permeability whichever the tested dietary group. Hence, as no channels accounted for glycerol transport and mainly the lipid pathway was being used, any difference in membrane fluidity may lead to increased bilayer permeability to glycerol. Higher $P_{g l y}$ relative to control with no change in $E_{a}$ was detected for the dietary group fed a mixture of CLA isomers, suggesting an effect on lipid bilayer fluidity rather than on the protein channel. In separate, $c 9, t 11$ and $t 10, c 12$ CLA isomers did not significantly alter the glycerol permeability, but together, they potentiate a significant effect.

The fatty acid profile of membrane vesicles showed only slight differences among dietary groups for the partial sums of SFA, MUFA and natural PUFA. On the contrary, CLA isomers incorporation into membranes was distinct among all groups. It has been suggested that both major CLA isomers specifically replace the essential fatty acids arachidonic (C20:4n-6) and linoleic (C18:2n6) into membrane phospholipids [5], but our results do not support this finding (Table 1). In our work, CLA isomeric distribution in membrane vesicles was not proportional to its correspondent percentages added to the diet. As expected, membranes from the mixture group (M) fed $1 \%$ of combined isomers $c 9, t 11$ and $t 10, c 12$ were the richest in CLA, showing also higher proportions of the minor isomers. It has been reported that incorporation of unsaturated fats into cellular membranes increases membrane fluidity and permeability $[25,26]$. The greatest increase in fluidity occurs with the addition of 2 and 3 double bonds, that allow considerable bending in the fatty acid chain thus inducing a decrease in the average chain length [27]. A recent review compiles results from a battery of biophysical techniques demonstrating that the insertion of docosahexaenoic acid (DHA; C22:4n-6) into membranes creates liquid disordered domains containing DHA-phospholipids and depleted in cholesterol, having looser lipid packing, thereby increasing membrane fluidity [28]. Our results suggest that the c9,t11 isomer intake promotes a higher incorporation of the $\mathrm{t} 10, \mathrm{c} 12$ in the membrane, thereby increasing the final CLA amount (group M, Table 1). In addition to total CLA amount, the diversity of isomers found in the mixture $M$ group may alter fatty acids arrangement and their packing in the bilayer changing membrane fluidity, thus explaining the observed increase in glycerol permeability. An increase in bilayer permeability has also been reported for liposomes containing CLA isomers [29].

In conclusion, this study suggests that a palm oil-based diet supplemented with a mixture of $c 9, t 11$ and $t 10, c 12$ CLA isomers in Wistar rats, in contrast to the individual isomers, positively affects the glycerol membrane permeability of kidney proximal tubule, possibly by means of an increase in lipid bilayer fluidity. This fact may be of biological relevance since in the kidney, glycerol can be either metabolized in situ or converted to glucose in 
the liver. Conversely, the data indicates that CLA isomers, isolated or as a mixture, do not affect aquaporin-mediated water transport.

\section{Acknowledgments}

This study was supported by FCT Grants POCTI/CVT/2002/ 44750 and PTDC/CVT/2006/66114 and individual FCT fellowships to Susana Martins (SFRH/BD/2005/22566) and Paula Alexandra Lopes (SFRH/BPD/2005/23931). The authors acknowledge Ana Madeira for technical support. We also thank Susana Alves and Rui Bessa (Unidade de Produção Animal, L-INIA-REQUIMTE) for providing fatty acid profile analyses.

\section{References}

[1] A. Bhattacharya, J. Banu, M. Rahman, J. Causey, G. Fernandes, Biological effects of conjugated linoleic acids in health and disease, J. Nutr. Biochem. 17 (2006) 789-810.

[2] S.V. Martins, P.A. Lopes, C.M. Alfaia, V.S. Ribeiro, T.V. Guerreiro, C.M. Fontes, M.F. Castro, G. Soveral, J.A. Prates, Contents of conjugated linoleic acid isomers in ruminant-derived foods and estimation of their contribution to daily intake in Portugal, Br. J. Nutr. 98 (2007) 1206-1213.

[3] M. Plourde, S. Jew, S.C. Cunnane, P.J. Jones, Conjugated linoleic acids: why the discrepancy between animal and human studies?, Nutr Rev. 66 (2008) 415421.

[4] J. Salas-Salvado, F. Marquez-Sandoval, M. Bullo, Conjugated linoleic acid intake in humans: a systematic review focusing on its effect on body composition, glucose, and lipid metabolism, Crit. Rev. Food Sci. Nutr. 46 (2006) 479-488.

[5] C.J. Field, P.D. Schley, Evidence for potential mechanisms for the effect of conjugated linoleic acid on tumor metabolism and immune function: lessons from n-3 fatty acids, Am. J. Clin. Nutr. 79 (2004) 1190S-1198S.

[6] K. Ishibashi, M. Kuwahara, Y. Gu, Y. Kageyama, A. Tohsaka, F. Suzuki, F. Marumo, S. Sasaki, Cloning and functional expression of a new water channe abundantly expressed in the testis permeable to water, glycerol, and urea, J. Biol. Chem. 272 (1997) 20782-20786.

[7] H. Kuriyama, S. Kawamoto, N. Ishida, I. Ohno, S. Mita, Y. Matsuzawa, K. Matsubara, K. Okubo, Molecular cloning and expression of a novel human aquaporin from adipose tissue with glycerol permeability, Biochem. Biophys. Res. Commun. 241 (1997) 53-58.

[8] E. Sohara, T. Rai, J. Miyazaki, A.S. Verkman, S. Sasaki, S. Uchida, Defective water and glycerol transport in the proximal tubules of AQP7 knockout mice, Am. J. Physiol. Renal Physiol. 289 (2005) F1195-F1200.

[9] D.O. Edem, Palm oil: biochemical, physiological, nutritional, hematological, and toxicological aspects: a review, Plant Foods Hum. Nutr. 57 (2002) 319341.

[10] P.A. Lopes, S.V. Martins, M.S. Pinho, C.M. Alfaia, C.M. Fontes, P.O. Rodrigues, G.S. Morais, M.F. Castro, R. Pinto, J.A. Prates, Diet supplementation with the cis9, trans-11 conjugated linoleic acid isomer affects the size of adipocytes in Wistar rats, Nutr. Res. 28 (2008) 480-486.
[11] G. Soveral, R.I. Macey, T.F. Moura, Water permeability of brush border membrane vesicles from kidney proximal tubule, J. Membr. Biol. 158 (1997) 219-228.

[12] G. Soveral, R.I. Macey, T.F. Moura, Mechanical properties of brush border membrane vesicles from kidney proximal tubule, J. Membr. Biol. 158 (1997) 209-217.

[13] M.M. Bradford, A rapid and sensitive method for the quantitation of microgram quantities of protein utilizing the principle of protein-dye binding, Anal. Biochem. 72 (1976) 248-254.

[14] G. Perevucnik, P. Schurtenberger, D.D. Lasic, H. Hauser, Size analysis of biological membrane vesicles by gel filtration, dynamic light scattering and electron microscopy, Biochim. Biophys. Acta 821 (1985) 169-173.

[15] M.P. van Heeswijk, C.H. van Os, Osmotic water permeabilities of brush border and basolateral membrane vesicles from rat renal cortex and small intestine, J. Membr. Biol. 92 (1986) 183-193.

[16] J.A. Dix, D.A. Ausiello, C.Y. Jung, A.S. Verkman, Target analysis studies of red cell water and urea transport, Biochim. Biophys. Acta 821 (1985) 243-252.

[17] K. Raes, V. Fievez, T.T. Chow, D. Ansorena, D. Demeyer, S. De Smet, Effect of diet and dietary fatty acids on the transformation and incorporation of C18 fatty acids in double-muscled Belgian Blue young bulls, J. Agric. Food Chem. 52 (2004) 6035-6041.

[18] O.A. Rego, H.J. Rosa, S.M. Regalo, S.P. Alves, C.M. Alfaia, J.A. Prates, C.M. Vouzela, R.J. Bessa, Seasonal changes of CLA isomers and other fatty acids of milk fat from grazing dairy herds in the Azores, J. Sci. Food Agric. 88 (2008) 1855-1859.

[19] M.T. Kramers, G.B. Robinson, Studies on the structure of the rabbit kidney brush border, Eur. J. Biochem. 99 (1979) 345-351.

[20] G.A. Quamme, Effect of parathyroid hormone and dietary phosphate on phosphate transport in renal outer cortical and outer medullary brush-border membrane vesicles, Biochim. Biophys. Acta 1024 (1990) 122-130.

[21] J.P. Quigley, G.S. Gotterer, Distribution of (Na+-K+)-stimulated ATPase activity in rat intestinal mucosa, Biochim. Biophys. Acta 173 (1969) 456-468.

[22] H. Murer, E. Ammann, J. Biber, U. Hopfer, The surface membrane of the small intestinal epithelial cell. I. Localization of adenyl cyclase, Biochim. Biophys. Acta 433 (1976) 509-519.

[23] R.E. Farmer, R.I. Macey, Perturbation of red cell volume: rectification of osmotic flow, Biochim. Biophys. Acta 196 (1970) 53-65.

[24] B. Yang, D. Zhao, A.S. Verkman, Evidence against functionally significant aquaporin expression in mitochondria, J. Biol. Chem. 281 (2006) 16202-16206.

[25] R.C. Poulsen, P.J. Moughan, M.C. Kruger, Long-chain polyunsaturated fatty acids and the regulation of bone metabolism, Exp. Biol. Med. (Maywood) 232 (2007) 1275-1288.

[26] W.G. Hill, E. Almasri, W.G. Ruiz, G. Apodaca, M.L. Zeidel, Water and solute permeability of rat lung caveolae: high permeabilities explained by acyl chain unsaturation, Am. J. Physiol. Cell Physiol. 289 (2005) C33-C41.

[27] W. Stillwell, S.R. Wassall, Docosahexaenoic acid: membrane properties of a unique fatty acid, Chem. Phys. Lipids 126 (2003) 1-27.

[28] S.R. Wassall, W. Stillwell, Docosahexaenoic acid domains: the ultimate nonraft membrane domain, Chem. Phys. Lipids 153 (2008) 57-63.

[29] J.J. Yin, J.K. Kramer, M.P. Yurawecz, A.R. Eynard, M.M. Mossoba, L. Yu, Effects of conjugated linoleic acid (CLA) isomers on oxygen diffusion-concentration products in liposomes and phospholipid solutions, J. Agric. Food Chem. 54 (2006) 7287-7293. 